\title{
Action of Nicotine upon the Blood Pressure in Rabbits, Deprived of the Coeliac and Superior Mesenteric Ganglia and the Suprarenals.
}

\author{
By \\ Masari Tiba. \\ (千莎 腾) \\ (From the Physiological Laboratory of Prof. F. Satake, \\ Tohoku Imperial University, Sendai.)
}

Pressoric effect of nicotine is not altered by depriving the epinephrine secretion from the suprarenal capsules or their removal. ${ }^{1)}$

In the hands of Hous sa $y$ and Molin elli, the pressoric effect was nullified by taking out the coeliac plexus besides the suprarenals. ${ }^{2}$

Gley was almost unable to bring about an elevation in the blood pressure in a dog by nicotine when the spinal cord was destroyed and the suprarenals were removed, while anagyrine, it was noted, acts to elevate the pressure though small. ${ }^{3}$ ) This experiment of Gley was not only seriously criticized by Stewart and Rogoff, but contradicted by an experiment of their own. ${ }^{\text {) }}$

In a decerebrated cat, Langle y was able to see a considerable rise in the blood pressure on giving nicotine, even after tying off the suprarenals from the circulation. ${ }^{5)}$ Hame t was able to see pressoric action of nicotine in the dog under chloralose even after removing the suprarenals and cutting the splanchnic nerves. ${ }^{6)}$

The present investigation has been carried out in order to test whether or not the exclusion of the coeliac plexi and the superior mesenteric

1) W a d a, Tohoku J. of Exp. Med., 1935, 25, 14. Literature there.

2) Hou s a y and Molinelli, Rev. Soc. Arg. de Biol., 1925, 1458 \& 482 ff., Mo 1 inelli, La secrecion de adrenalina, Buenos Aires 1926, $222 \& 239$ ff., Hous s a y and M o I inelli, Am. J. of Physiol., 1926, 76, 560 \& 571.

3) Gley, C. r. Acad. Sc., 1914, 158, 2008.

4) Stewart and Rog off, J. of Pharm. \& Exp. Ther., 1919, 13, $184 \mathrm{f} \&$ 220-222.

5) L a ngley, J. of Physiol., 1918 (-19), 52, 261.

6) Ha met, Arch. de Pharmacodyn. et de Thér., 1930, 38, 382. 
ganglion is capable of nullifying or largely reducing the powerful pressor effect of nicotine, which is not materially altered by double suprarenalectomy.

The coeliac ganglia and the superior mesenteric ganglion were extirpated per laparotomiam without narcosis, and one month or later the suprarenal capsules in two sittings through the lumbar way without narcosis. Then one month or more was allowed to elapse before the blood pressure experiment.

Double discontinuance of the splanchnic nerves or loss of the suprarenals themselves does not change the arterial blood pressure in the ordinary experimental conditions at all, if sufficient time is allowed to elapse for subsidence of the disturbances in the organism resulting from fastening, anaesthetization, opening the abdominal cavity, etc.?)

The rabbit was fastened on its back without narcosis, and a carotid artery was connected to a mercury manometer. Nicotine puris Merck, as $1: 500$ watery solution, was injected into a marginal vein of the ear, previously denervated, in doses of $0.5 \mathrm{mgrm}$ : or $1.0 \mathrm{mgrm}$. per kilo of body weight. Nicotine solution was freshly prepared at each case.

\section{REsults.}

$0.5 \mathrm{mgrm}$. per kilo was applied in 5-13 seconds to two normal rabbits and three operated rabbits. Slight convulsions occurred a few seconds later, and continued some ten seconds.

In normal rabbits: No. $\mathrm{C} 77$, the mean arterial pressure first descended from about $100 \mathrm{mms}$. Hg. to $70 \mathrm{mms}$. in 14 seconds, then rose to $162 \mathrm{mms}$. Hg. in 38 seconds after the start of injection (injection 6 seconds), afterwards it descended slowly, so that 5 minutes after the injection $96 \mathrm{mms}$. Hg. was recorded and followed by insignificant fluctuations. No. C 78, the pressure dropped from $106 \mathrm{mms}$. Hg. to $56 \mathrm{mms}$. in 8 seconds, then rose to $134 \mathrm{mms}$. $\mathrm{Hg}$. in 49 seconds from the end of injection (injection in 13 seconds), 5 minutes after the injection 112 mms., later it fluctuated between 100 and $110 \mathrm{mms}$. Hg.

In the animals operated on: No. C 52. From $126 \mathrm{mms}$. Hg. to 82 mms. in 17 seconds after the start of injection (injection in 11 seconds), and to $154 \mathrm{mms}$. Hg. in 30 seconds. In 5 minutes it went down to 114 mms.Hg., afterwards it fluctuated between 108 and $114 \mathrm{mms}$.Hg.for one hour, in the next hour it was somewhat low throughout ( $92 \mathrm{mms}$. Hg.).

7) Suzuki, Tohoku J. of Exp. Med., 1928, 12, 87. Literature there. About 10 days interval was noted in a recent paper as sufficient for recovering the blood pressure after double splanchnictomy: Horne, McDongall and Magee, J. of Physiol, 1933(-34), $80,56$. 


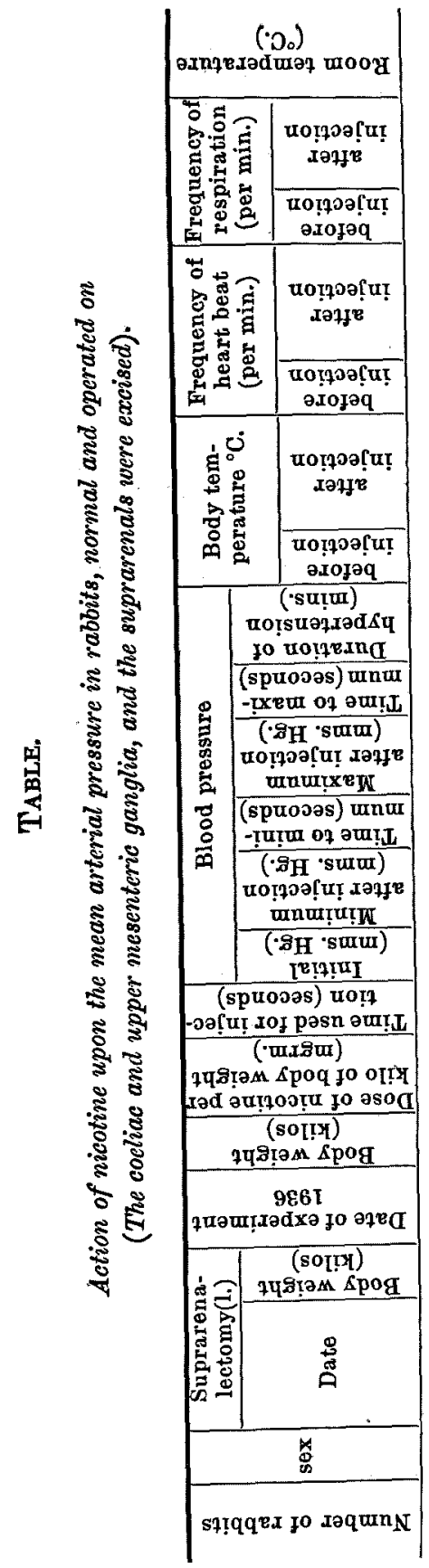

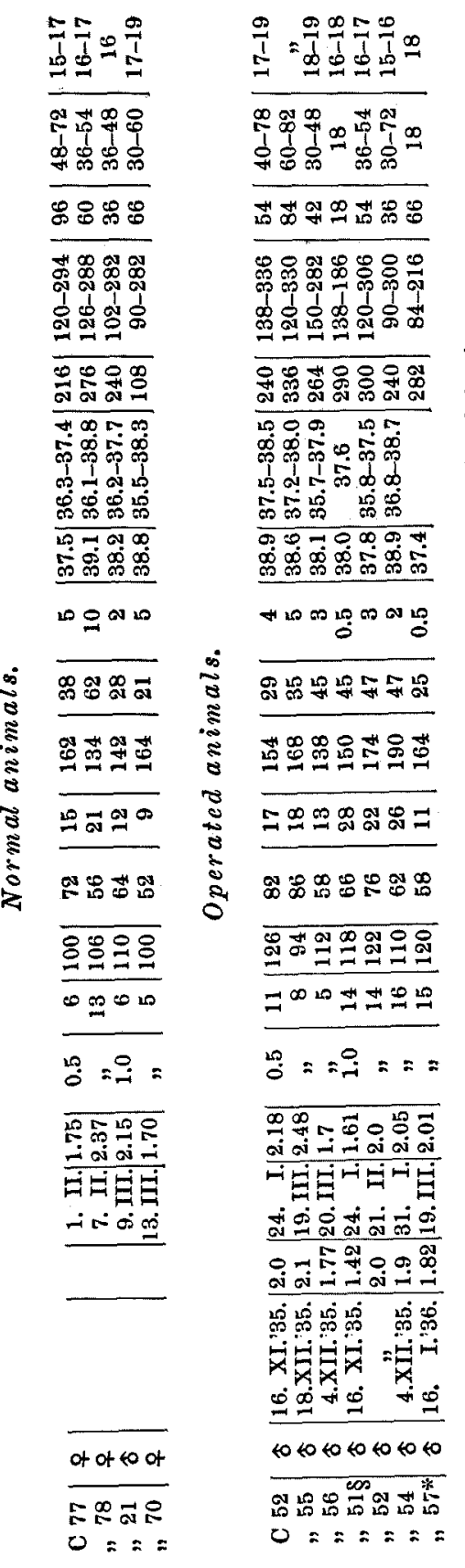


No. C 55. From $94 \mathrm{mms}$. Hg. to $86 \mathrm{mms}$. Hg. in 18 seconds after the start of injection (injection in 5 seconds), and to $168 \mathrm{mms}$. in 35 seconds. 5 minutes after the injection it was restored to the initial height and following that there were some fluctuation of from 100 to 92 mms. for two hours.

No. C 56. From $112 \mathrm{mms}$. Hg. to $58 \mathrm{mms}$. in 13 seconds after the start of injection (injection in 5 seconds), and to 138 in 45 seconds; 5 minutes after the injection it was as low as $72 \mathrm{mms}$., but afterwards it recovered the initial height (98-118 mms. Hg. for two hours).

$1 \mathrm{mgrm}$. nicotine per kilo was injected intravenously in 5-16 seconds in two normal and 4 operated animals. Convulsions were stronger and more prolonged than in the cases with $0.5 \mathrm{mgrm}$. per kilo. And two rabbits operated on died, one in 3 minutes, the other in 10 minutes after the injection, which harmonizes with the finding of Oika wa a), who compared the sensibility of the rabbits, indefinitely surviving double suprarenalectomy, against nicotine with the normal individuals. He injected the solution more slowly than these cases and several manipulations such as fastening the animal on the board, uncovering the carotid artery, were conducted in ours, while he held the animal as undisturbed as possible, so that the figures in both papers cannot be directly compared.

Normal rabbits: No. C 21, the mean arterial pressure went down on nicotine from $110 \mathrm{mms}$. $\mathrm{Hg}$. to $64 \mathrm{mms}$. $\mathrm{Hg}$. in 12 seconds after the start of injection (injection in 6 seconds), and up to $142 \mathrm{mms}$. $\mathrm{Hg}$. in 28 seconds, then it fell again a little (to $86 \mathrm{mms}$. $\mathrm{Hg}$.) and about half hour was needed to see a complete recovery, then the pressure remained practically unaltered for 1.5 hours.

No. C 70, similar changes occurred in this rabbit; $100 \mathrm{mms} . \mathrm{Hg}$. before the injection, $52 \mathrm{mms}$. $\mathrm{Hg}$. 9 seconds after the start of injection, $164 \mathrm{mms}$. Hg. 21 seconds after the start of injection. For about twenty minutes about $100 \mathrm{mms}$. Hg. was held, and afterwards it became a little high.

The operated rabbits: No. C 51, before $118 \mathrm{mms}$. Hg., 28 seconds after nicotine 66 mms. Hg., (injection in 14 seconds) 45 seconds 150 mms. Hg., afterwards the pressure fell rapidly and about ten minutes later the animal died.

No. C 52. $122 \mathrm{mms}$. Hg., 22 seconds after nicotine $76 \mathrm{mms}$. Hg., (injection in 14 seconds) 47 seconds $174 \mathrm{mms}$. $\mathrm{Hg} .5$ minutes later 110

8) Oikawa, Tohoku J. of Exp. Med., 1931, 18, 27. 
mms. Hg., thereafter this level was held for about one hour, then it became somewhat lower.

No. C 54. Initial pressure $110 \mathrm{mms}$. Hg., 26 seconds after nicotine (injection in 16 seconds) $62 \mathrm{mms}$. Hg., 47 seconds $190 \mathrm{mms}$. Hg., then it had a tendency of descending, 5 minutes after nicotine $50 \mathrm{mms} . \mathrm{Hg}$., 5 minutes further later it went up to about $90 \mathrm{mms}$. $\mathrm{Hg}$., followed by a small elevation; 1.5 hours after the injection the pressure was a little lower.
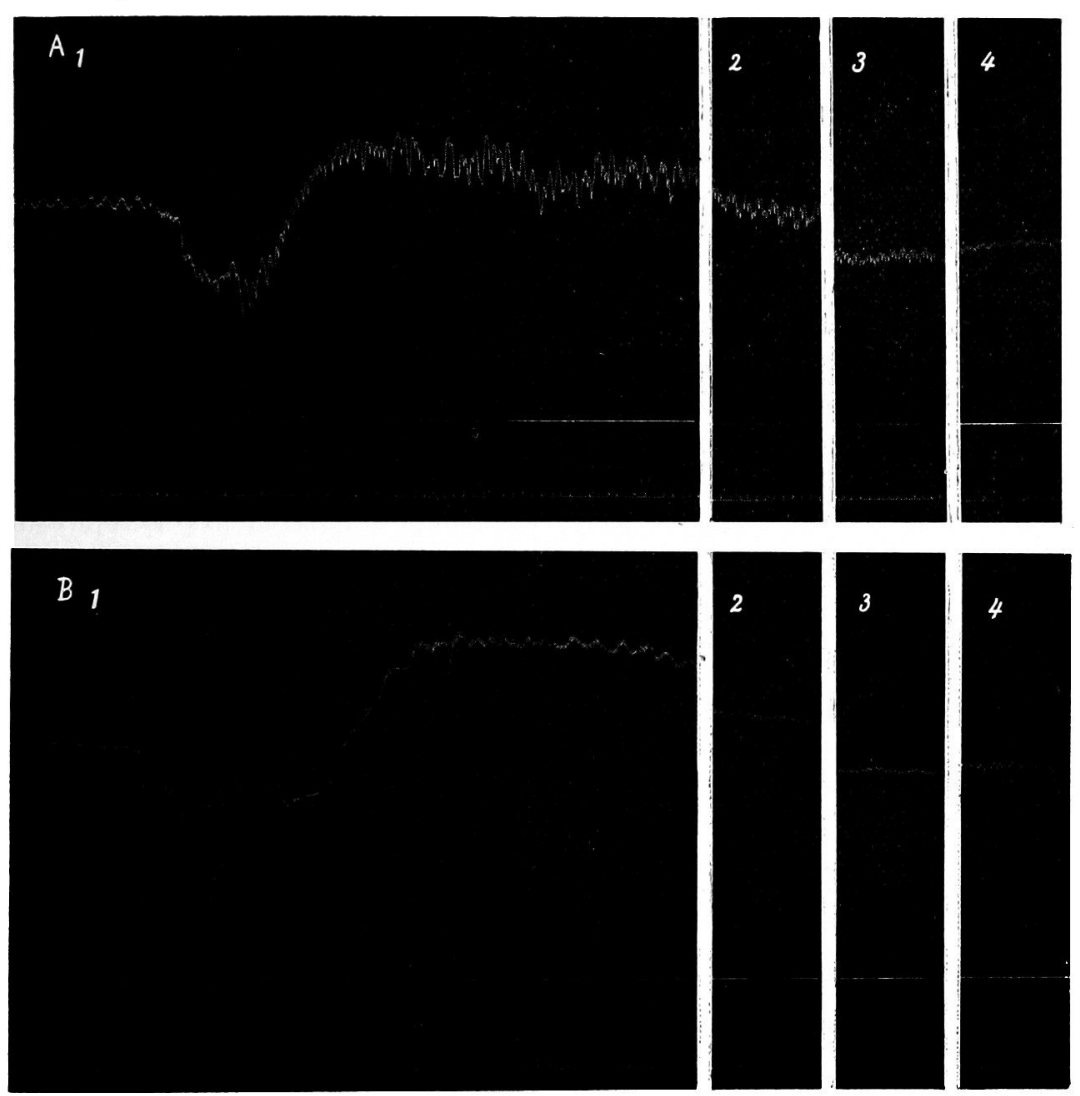

Showing influence of intravenous injection of nicotine ( 1 mgrm. per kilo of body weight) on the blood pressure of the normal rabbit and that surviving removal of the suprarenals and the coeliac and superior mesenteric ganglia.

Reduced to $\frac{2}{5}$. A : Normal rabbit (No. 21). $\delta$ 2.15 kilos. 9. III. 1936. B : Operated rabbit (No. 52). क 2.0 kilos. 21. II. 1936.

Upper curve: blood pressure by $\mathrm{Hg}$-manometer; middle line : zero line for blood pressure; lower line: time in seconds. Section 2 is two and half minutes after injection; Section 3, five minutes after injection; Section 4, ten minutes after injection. 
No. C 57, from $128 \mathrm{mms}$. Hg. the pressure went down to $58 \mathrm{mms}$. $\mathrm{Hg} .11$ seconds after nicotine (injection in 5 seconds), then up to 164 mms. $\mathrm{Hg} .25$ seconds after nicotine, then descended rapidly $50 \mathrm{mms}$. $\mathrm{Hg}$. 60 seconds and $28 \mathrm{mms}$. Hg. 2 minutes after the injection, then shortly afterwards the animal died.

The blood pressure tracings from one of both series of rabbits, receiving $1 \mathrm{mgrm}$. nicotine per kilo are above reproduced.

From the data above stated it may be acknowledged that the rabbits indefinitely surviving the removal of the suprarenals and the coeliac and superior mesenteric ganglia do not differ from the normal in respect to the vascular reaction against nicotine, especially the magnitude of the blood pressure fall occurring immediately on an intravenous injection of nicotine and that of the subsequent pressure elevation, and the time when they occur. The experiment of Houssay and Molinelli was acute in nature. ${ }^{2)}$

The strong pressure elevation was followed of ten by a somewhat large, but slow fall in the animals operated on.

The normal rabbits showed a bradycardia some minutes after nicotine; this was remarkable in the rabbits operated on, only when the poisoning was tọo strong as to cause death in some minutes. But this difference I should not extend to generalize, since the tracing was not carried out continuously except some minutes following the injection.

The body temperature fall of similar degree occurred in both the sets of animals, normal and operated on.

\section{SumMary.}

Nicotine was intravenously administered into normal rabbits, and rabbits indefinitely surviving removal of the suprarenals, and the coeliac and upper mesenteric ganglia, in doses of $0.5 \mathrm{mgrm}$. and $1 \mathrm{mgrm}$. per kilo of body weight with the mean blood pressure recording.

The removal does not alter at all the magnitude of the pressure fall occurring immediately after the nicotine injection and that of the subsequent pressure elevation. Neither is the time when they occur altered.

The low resistance of the animals so operated on, though they-were indefinitely well surviving, against the drug, has been also proved here. 